PAPER

\section{Theory of the thickness dependence of the charge density wave transition in $1 \mathrm{~T}-\mathrm{TiTe}_{2}$}

To cite this article: Jianqiang Sky Zhou et al 2020 2D Mater. 7045032

View the article online for updates and enhancements.
You may also like

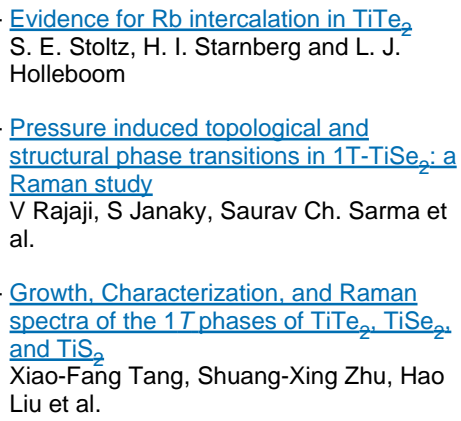

Growth, Characterization, and Raman spectra of the $1 T$ phases of $\mathrm{TiTe}_{2}, \mathrm{TiSe}_{2^{2}}$ and TiS

Xiao-Fang Tang, Shuang-Xing Zhu, Hao Liu et al.

\section{Recent citations}

- Gaussian time-dependent variational
$\frac{\text { principle for the finite-temperature }}{\text { anharmonic lattice dynamics }}$
Jae-Mo Linm and Cheol-Hwan Park
- The stochastic self-consistent harmonic
$\frac{\text { approximation: calculating vibrational }}{\text { properties of materials with full quantum }}$
$\frac{\text { and anharmonic effects }}{\text { Lorenzo Monacelli et al }}$
- Temperature evolution of quasiparticle
$\frac{\text { dispersion and dynamics in semimetallic }}{1 T T i T e 2 \text { via high-resolution angle-resolved }}$
$\frac{\text { photoemission spectroscopy and ultrafast }}{\text { optical pump-probe spectroscopy }}$
Shuang-Xing Zhu et al




\section{D Materials}

PAPER

CrossMark

\section{Theory of the thickness dependence of the charge density wave transition in 1 T-TiTe 2}

8 April 2020

REVISED

2 August 2020

ACCEPTED FOR PUBLICATION

12 August 2020

PUBLISHED

30 September 2020

\author{
Jianqiang Sky Zhou ${ }^{1}$, Raffaello Bianco ${ }^{2}$, Lorenzo Monacelli ${ }^{3}$, Ion Errea ${ }^{2,4,5}$, Francesco Mauri ${ }^{3,6}$ \\ and Matteo Calandra ${ }^{1,6,7}$ (D) \\ 1 Sorbonne Université, CNRS, Institut des Nanosciences de Paris, UMR7588, F-75252, Paris, France \\ 2 Centro de Física de Materiales (CSIC-UPV/EHU), Manuel de Lardizabal pasealekua 5, 20018 Donostia-San Sebastián, Basque Country, \\ Spain \\ 3 Dipartimento di Fisica, Università di Roma La Sapienza, Piazzale Aldo Moro 5, I-00185 Roma, Italy \\ 4 Fisika Aplikatua 1 Saila, Gipuzkoako Ingeniaritza Eskola, University of the Basque Country (UPV/EHU), Europa Plaza 1, 20018, \\ Donostia San Sebastián, Basque Country, Spain \\ 5 Donostia International Physics Center (DIPC), Manuel de Lardizabal pasealekua 4, 20018 Donostia San Sebastián, Basque Country, \\ Spain \\ 6 Graphene Labs, Fondazione Istituto Italiano di Tecnologia, Via Morego, I-16163 Genova, Italy \\ 7 Department of Physics, University of Trento, Via Sommarive 14, 38123 Povo, Italy \\ E-mail: matteo.calandra@upmc.fr
}

Keywords: transition metal dichalcogenide, bulk and monolayer tite ${ }_{2}$, charge density wave, anharmoncity, thickness dependence, phonons

\section{Introduction}

Charge density waves (CDWs) are ubiquitous phenomena in condensed matter physics as they appear in many systems having different electronic structures and dimensionalities. While lots of work has been carried out in one-dimensional (1D) systems with very sharp Fermi surfaces, the mechanism generating charge ordering in higher dimension is still controversial, mainly because Fermi surfaces are composed of multiple sheets and are not point-like, as in 1D. Furthermore, the variability of the electronic and structural properties substantially affects the interplay of the three fundamental interactions competing in CDW formation: electronelectron, electron-phonon and anharmonicity. These factors complicate the explanation of the mechanism responsible for charge ordering and how the latter is affected by external perturbations.

Metallic and semimetallic transition metal dichalcogenides (TMDs) with chemical formula $\mathrm{TX}_{2}$, where $\mathrm{T}$ is a transition metal and $\mathrm{X}$ is a chalcogene, are among the first 3D systems where CDWs were detected and are an example of this large variability as several different ordering vectors and reconstructions can be found by weakly perturbing the chemical (doping) or structural (e.g. stacking or polytype variation) properties [1]. The advent of mechanical exfoliation and the synthesis of 2D crystals [2] added one additional parameter, namely the sample thickness, that can be made as thin as that of a $\mathrm{TX}_{2}$ single layer. It becomes then possible to study the CDW 
crossover from the bulk to the $2 \mathrm{D}$ case. However, up to now, in most of the cases the single layer and bulk display similar ordering vectors and qualitatively similar charge density wave patterns (although the charge density wave critical temperature, $\mathrm{T}_{\mathrm{CDW}}$, can differ in the same compound depending on the thickness of the sample). The $2 \mathrm{H}$ polytypes such as $2 \mathrm{H}-\mathrm{NbSe}_{2}$, $2 \mathrm{H}-\mathrm{TaS}_{2}$ and $2 \mathrm{H}-\mathrm{TaSe}_{2}$, display CDW with the same periodicity in bulk and single-layer form. On the contrary, $2 \mathrm{H}-\mathrm{NbS}_{2}$ does not show evidence of charge ordering in bulk, while contradictory experimental results have been reported in supported single layers, as $1 \mathrm{H}-\mathrm{NbS}_{2}$ (the notation $1 \mathrm{H}$ meaning a single-layer H polytype) on $\mathrm{Au}(111)$ does not display a CDW [3], while $1 \mathrm{H}-\mathrm{NbS}_{2}$ on $6 \mathrm{H}-\mathrm{SiC}(0001)$ endures a $3 \times 3$ reconstruction [4]. The $1 \mathrm{~T}$ polytypes display fairly similar behaviour. $1 \mathrm{~T}-\mathrm{TiSe}_{2}$ undergoes a CDW with the same in-plane periodicity both in bulk and single layer, despite some differences in $\mathrm{T}_{\mathrm{CDW}}$ depending on the substrate or the doping level [5-11]. $1 \mathrm{~T}$ $\mathrm{TaS}_{2}$ shows a David star $\sqrt{13} \times \sqrt{13}$ reconstruction both in bulk and in single layer [12] (in the bulk the $3 \mathrm{D}$ stacking of the stars makes the understanding more complicate and controversial). Finally, $1 \mathrm{~T}-\mathrm{VSe}_{2}$ reconstructs with a $4 \times 4$ periodicity both in bulk and in single layer with a non-monotonic dependence of $\mathrm{T}_{\mathrm{CDW}}$ as a function of layer number [13].

In this respect, the case of $1 \mathrm{~T}-\mathrm{TiTe}_{2}$ is definitely surprising and deserves particular scrutiny. Singlelayer $1 \mathrm{~T}$ - $\mathrm{TiTe}_{2}$ displays a $2 \times 2 \mathrm{CDW}$ with $\mathrm{T}_{\mathrm{CDW}}=92$ $\mathrm{K}$, but absolutely no CDW occurs in bulk [14]. This result is even more puzzling given the similarity of the electronic structure of $1 \mathrm{~T}-\mathrm{TiTe}_{2}$ bulk/monolayer with that of $1 \mathrm{~T}-\mathrm{TiSe}_{2}$ bulk/monolayer, the latter undergoing a CDW with the same ordering vector at all thicknesses. Harmonic density functional perturbation theory calculations with gradient corrected functionals are unable to explain the main experimental facts, as under this approximation no CDW occurs in $\mathrm{TiTe}_{2}$ neither in bulk nor single layer [14]. A recent theoretical work by Guster et al [15] claimed that the CDW in single layer could be either due to strain or induced by the exchange interaction. Substrate strain is an unlikely explanation as on the experimental side $\mathrm{TiTe}_{2}$ one-layer thick films are deposed on an incommensurate substrate and the measured lattice parameter is practically the same as in the bulk. Moreover, theory showed that large strains are needed to induce the CDW [15], a result recently confirmed by strongly epitaxially strained $\mathrm{TiTe}_{2}$ flakes of thickness up to $32 \mathrm{~nm}$ on InAs(111)/Si(111) substrates [16].

Hartree-Fock exchange is then a plausible explanation, given its importance in bulk and single-layer $1 \mathrm{~T}^{-\mathrm{TiSe}_{2}}[17,18]$. This conclusion is also supported by finite difference harmonic calculations with the HSE06 functional finding the occurrence of the most unstable phonon mode at the $\mathrm{M}$ point of the Brillouin zone (BZ) compatible with a $2 \times 2$ reconstruction, in agreement with experiments [15]. However, as we will show here, harmonic calculations based on the HSE06 functional predict the occurrence of CDW both in single-layer and bulk $1 \mathrm{~T}^{-\mathrm{TiTe}_{2}}$, in clear disagreement with experiments. Therefore, calculations in literature are unable to explain the reduction of CDW in $1 \mathrm{~T}-\mathrm{TiTe}_{2}$ as a function of layer thickness.

In this work, we study the vibrational properties of suspended single-layer and bulk $1 \mathrm{~T}$ $\mathrm{TiTe}_{2}$, by accounting for non-perturbative anharmonicity within the stochastic self-consistent harmonic approximation (SSCHA) [19-22]. The SSCHA is a stochastic variational technique developed by the authors that allows to access the non-perturbative quantum anharmonic free energy and its second derivative (i.e. the phonon spectra) from the evaluation of forces on supercells with atoms displaced from their equilibrium positions following a suitably chosen Gaussian distribution. The forces can be evaluated by using any force engine. We show that the interplay between non-perturbative anharmonicity and exchange renormalization of the electronphonon coupling explains the thickness dependence of CDW in $1 \mathrm{~T}-\mathrm{TiTe}_{2}$. Moreover, we completely characterize the electronic and vibrational properties of the $2 \times 2$ reconstruction in single-layer $1 \mathrm{~T}-\mathrm{TiTe}_{2}$.

\section{Computational details}

Density-functional theory (DFT) calculations using the Perdew-Burke-Ernzerhof (PBE) [23] and HSE06 [24, 25] exchange-correlation functionals are carried out using the CRYSTAL [26] and the Quantum-ESPRESSO [27, 28] packages. The optimized lattice parameters for the undistorted $\mathrm{CdI}_{2}$ phase of bulk and single-layer $\mathrm{TiTe}_{2}$ can be found in table 1. As it can be seen, both HSE06 and PBE accurately describe the in-plane lattice parameter but substantially overestimate the interlayer distance in the bulk, due to the lack of Van der Waals forces. A practical and common way to avoid this problem in TMDs is to adopt the experimental measured lattice parameters $a=3.777 \AA$ and for the bulk, with $c=6.495 \AA$ [14]. In the single-layer case, we used a $12.99 \AA$ vacuum region to avoid interactions between periodic images. We perform geometrical optimization of internal coordinates. For the CRYSTAL code we use an allelectron molecular def2-TZVP basis set [29] reoptimized for solid state calculations [17] for the Ti atom and a pVDZ-PP basis set for the Te atom [30, 31]. For Quantum-ESPRESSO HSE06 fully-relativistic calculations, we used norm-conserving ONCV pseudopotentials from the Pseudo-dojo library [32] (high accuracy). The electronic and harmonic phonon bands are calculated using the same $\Gamma$ centered $\mathbf{k}$ points mesh and electronic temperature (i.e. smearing in Fermi-Dirac function, see table $\mathrm{A} 1$ in appendix A for more technical details). The $\mathbf{k}$-points mesh is rescaled according to the size of supercells (e.g. a $36 \times 36 \times 1 \mathbf{k}$-points mesh in $1 \times 1 \times 1$ cell becomes 
Table 1. Completely optimized structural parameters for bulk and single-layer $\mathrm{TiTe}_{2}$ in the undistorted $\mathrm{CdI}_{2}$ phase with space group $\mathrm{P} \overline{3} \mathrm{ml}$ (number 164) compared to experiments. The quantity $z_{\mathrm{Te}}$ is the only internal parameter not determined by symmetry, namely the tellurium distance from the plane of Ti atoms. The $z_{\mathrm{Te}}$ values in brackets are obtained assuming the experimental lattice parameters $a=3.777 \AA$ and $c=6.495 \AA$ and optimizing only internal coordinates. We use the experimental lattice parameters and the values of $z_{\mathrm{Te}}$ in brackets in all bands and phonon calculations.

\begin{tabular}{lcccc}
\hline \multicolumn{5}{c}{ bulk } \\
\hline & PBE & HSE06 & EXP [14] & EXP [33] \\
\hline$a$ & 3.77 & 3.79 & 3.777 & 3.768 \\
$c$ & 6.94 & 6.94 & 6.495 & 6.524 \\
$z_{\mathrm{Te}}$ & $1.73(1.71)$ & $1.69(1.68)$ & & 1.66 \\
\hline
\end{tabular}

\begin{tabular}{lccc}
\hline & \multicolumn{3}{c}{ monolayer } \\
\hline$a$ & 3.765 & 3.788 & 3.78 \\
$z_{\mathrm{Te}}$ & $1.74(1.73)$ & $1.69(1.70)$ & \\
\hline
\end{tabular}

$9 \times 9 \times 1$ in a $4 \times 4 \times 1$ cell). Since the harmonic phonon frequency is very sensitive to the chosen $\mathbf{k}$ points sampling and electronic temperature $\mathrm{T}_{e}$, the convergence of the lowest phonon frequency with respect to the k-points sampling and electronic temperature is carefully investigated for both DFT functionals (see figure A1 in appendix A). The HSE06 forces needed for the stochastic self-consistent harmonic approximation (SSCHA) [19-22] are computed with the CRYSTAL code.

\section{Results and discussion}

\subsection{Electronic structure of the undistorted $\mathrm{CdI}_{2}$ phase}

3.1.1. Theory.

The electronic structure of the undistorted $\mathrm{CdI}_{2}$ phase calculated from two DFT functionals, the scalar relativistic semilocal PBE and the scalar relativistic hybrid HSE06, is shown in figures 1 (a) and (b) for the monolayer and bulk, respectively. Both approximations give a semimetallic ground state, in agreement with several previous first-principles band structure calculations [15, 34-36]. The semimetallic character is due to the band overlap between the Te hole pockets at zone center (multiband in nature) and the Ti $3 d$ electron-pocketcat $\mathrm{M}$ in the monolayer at $\mathrm{L}$ in the bulk. In the bulk the band-overlap between $\mathrm{L}$ and $\Gamma$ is larger than for the single-layer case between $M$ and $\Gamma$. The inclusion of screened exchange within the HSE06 functional has two main effects: (i) to reduce the band-overlap between zone center and $\mathrm{M}(\mathrm{L})$ in the single layer (bulk) and (ii) to substantially increase the Fermi velocity of the Te bands close to zone center.

The inclusion of relativistic effects has minor consequences for the single layer, as shown in appendix $B$ for the PBE semilocal functional. In the bulk, it affects mainly the bands at zone center where there are three Te bands forming three hole pockets. In the absence of spin-orbit coupling (SOC), two of these bands (arising from Te $p$ orbitals) are degenerate at $\Gamma$, while the third one is not. SOC splits the two Te degenerate bands at zone center, downshifting one of the two and upshifting the other, as shown in figure 1 (c) for the HSE06 case and in appendix B for the PBE case, respectively. However, while in PBE the electron-electron interaction still leaves a portion of the lower of the two bands unoccupied, the combined effect of HSE06 and SOC leads to a completely occupied Te band at zone center (see figure 1 (c)). As we will show in the next paragraph, the combined effect of exchange and relativistic effects is needed to solve a long standing controversy in ARPES spectra.

\subsubsection{Comparison with ARPES.}

ARPES experiments show a semimetallic nature for both single-layer and bulk $\operatorname{TiTe}_{2}[14,34,37]$ in its $\mathrm{CdI}_{2}$ undistorted phase. The comparison between the calculated electronic structures and ARPES data for the single layer and bulk are shown in figure $1(\mathrm{~d}, \mathrm{e}, \mathrm{f})$. The HSE06 electronic structure and ARPES data are in excellent agreement for the single-layer case (panel d). The PBE approximation gives unrealistically too low Fermi velocities for the Te band close to zone center forming the largest hole pocket and a too large occupation of the Ti $3 d$ pocket at $\mathrm{M}$.

In the bulk case, ARPES spectra of $\mathrm{TiTe}_{2}$ have been measured in several works [14, 34, 37], we compare here with the most recent work of [14]. Some care is needed in comparing theory and experiments in bulk due to the very strong $k_{z}$ band dispersion and the fact that $k_{z}$ is not a good quantum number in ARPES. It is then not obvious that measurements really probe the bands at $k_{z}=0$. This issue has been carefully addressed in reference [34, 37], where the magnitude of $k_{z}$ dispersion along ML was estimated to be included between 20 and 100 meV binding energy. Experimentally, the determination of $k_{z}$ is complicated by the presence of what are usually labeled as non-free electrons final state effects (i.e. the approximation that the final-state surface-perpendicular dispersion $\epsilon_{\mathbf{k}_{\perp}}$ is assumed to be parabolic breaks down) [37]. For this reason theoretical description of ARPES for bulk $\mathrm{TiTe}_{2}$ is challenging.

In figure 1 panels $(e, f)$ we superimpose the bands along AL and along $\Gamma \mathrm{M}$ (plotted with different colors) to the experimental ARPES data. At the $\bar{M}$ point, ARPES would be consistent with the calculated electronic structure at a $k_{z}$ somewhere close to half the distance between $\Gamma$ and $M$.

At zone center, four or five bands are measured in ARPES, as it can be seen in figure 1(d) or in figure 3 of reference [14] panel (a). However, only three bands occur close to $\Gamma$ in the calculation, thus part of these bands are necessarily related to other values of $k_{z}$. As it can be seen from figure $1(\mathrm{~d}, \mathrm{e})$, this 


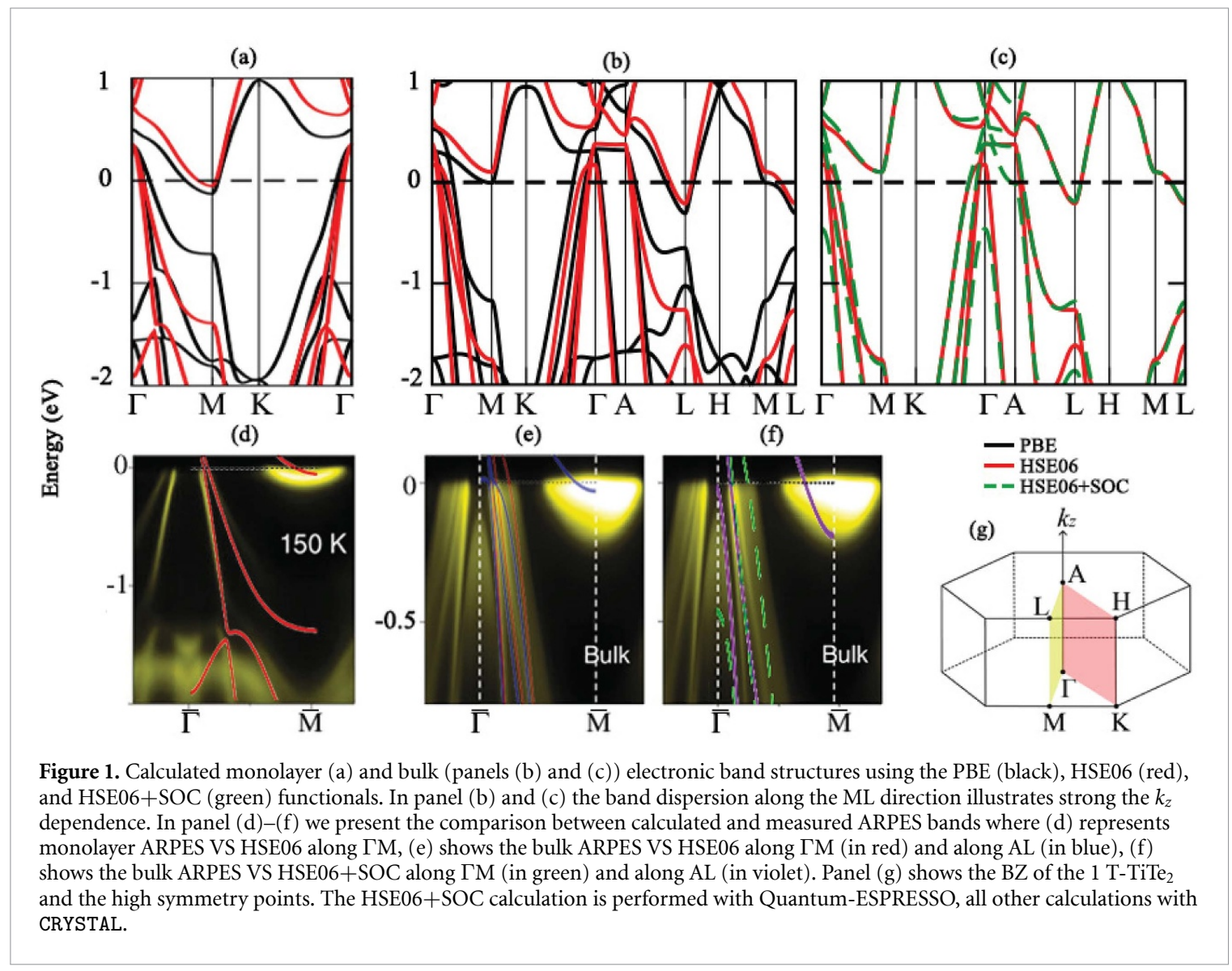

is consistent with the calculated band structure and its $k_{z}$ dispersion that generates more shadow bands. The non-relativistic HSE06 bands (panel d) are in better agreement with experiments if contributions from scattering at $k_{z} \neq 0$ are assumed to occur. Still, if relativistic effects are neglected, there is one important disagreement between theory and experiments, namely the fact that ARPES data show the presence of a completely occupied parabolic band at $\approx-0.135$ $\mathrm{eV}$ binding energy (see experimental data in figure 1 $(d, e))$ that is missing in all samples below three layers (see figure 3 of reference [14] panel (a)) and in all PBE calculations (including or neglecting SOC) for any value of $k_{z}$. This band was detected as a very broad feature in previous experimental ARPES work [34] (hatched region in figures 5, 13, and 14), however, its origin is unclear in literature. It was proposed to be due to many body effects beyond the single particle approximation.

Here we demonstrate, on the contrary, that this band arises from the combined effect of screened exchange and relativistic effects, as shown in figure 1, panel ( $\mathrm{f}$ ), as discussed in the previous subsection. In the HSE06 relativistic calculation the band is somewhat lower in energy at zone center than in experiments. This is most likely due to the $n$-doping occurring in $\mathrm{TiTe}_{2}$ samples. We also stress that the exact position of this band is extremely sensitive on the Te distance from the plane and differences of 0.01
$\AA$ leads to a sizeable energy shift. Thus our calculation explains this feature without invoking any many body effects and solves the long standing ARPES controversy.

\subsection{Harmonic phonon dispersion for the CdI 2 undistorted phase}

The harmonic phonon dispersion for bulk and single layer using the PBE and HSE06 functionals with and without SOC are shown in figure 2. The PBE phonon dispersion neglecting SOC are found to be in good agreement with previous calculations of references $[14,15]$ and show only positive phonon frequencies and no CDW formation, neither in bulk nor in monolayer. The inclusion of SOC leads to negligible differences both in bulk and monolayer. This means that the changes in the electronic structure due to the relativistic effects have only marginal consequences for the CDW formation.

The single-layer and bulk PBE phonon dispersion are, however, markedly different as in the former case a softening occurs at the M point, while no softening is seen in the bulk at the $\mathrm{L}$ point. The result changes completely if the HSE06 functional is used, as now both the bulk and the single layer show imaginary phonon frequencies (depicted as negative) at the $\mathrm{L}$ and $M$ points, respectively. Thus, within HSE06 and at the harmonic level, both single layer and bulk do display a CDW, in disagreement with experimental 


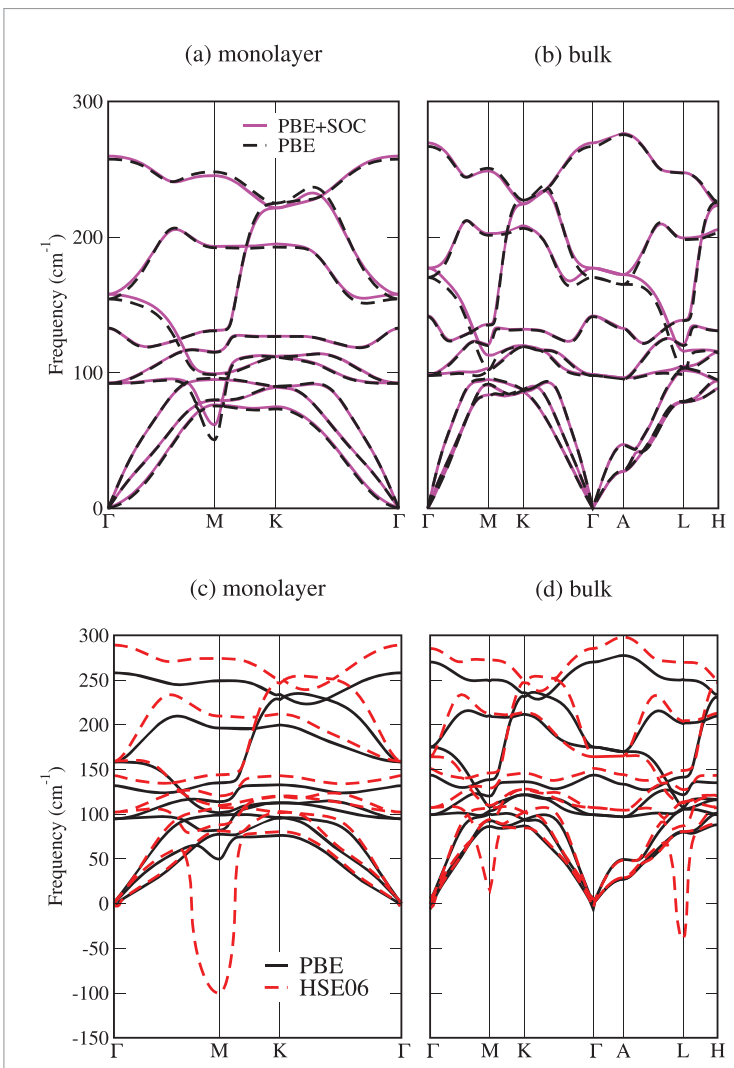

Figure 2. Top panels: the monolayer and bulk harmonic phonon dispersion in PBE with and without spin orbit coupling calculated with the QUANTUM-ESPRESSO code. Bottom panels: PBE and HES06 phonon dispersion neglecting spin-orbit coupling calculated with the CRYSTAL code.

data showing absence of charge ordering in the bulk. Therefore, the exchange interaction alone is not sufficient to explain the thickness dependence of the CDW in $\mathrm{TiTe}_{2}$, as previously proposed [15]. In the case of the single layer, we also calculated the energy gain by the distortion in a $2 \times 2$ supercell by displacing the atoms along the phonon pattern of the structural distortion, finding it to be approximately $3.13 \mathrm{meV}$ per Ti atom, a value in excellent agreement with the HSE06 plane waves calculation carried out in reference [15].

The different behaviour of the harmonic phonon dispersion at the L (bulk) and M (single layer) points for the different functionals can be due to two effects: (i) differences in the electronic structure and (ii) differences in the electron-phonon matrix elements. The softening is indeed due to the real part of the electronphonon self-energy phonon [38]:

$$
\prod_{\nu}^{\prime}(\mathbf{q})=\mathcal{P} \frac{1}{N_{k}} \sum_{\mathbf{k}, n m} \frac{\left|g_{\mathbf{k} n, \mathbf{k}+\mathbf{q} m}^{\nu}\right|^{2}\left(f_{\mathbf{k} n}-f_{\mathbf{k}+\mathbf{q} m}\right)}{\epsilon_{\mathbf{k} n}-\epsilon_{\mathbf{k}+\mathbf{q} m}}
$$

where $\mathcal{P}$ label the principal part, $N_{k}$ the number of $\mathbf{k}$ points used in the calculations $(120 \times 120$ and $80 \times 80 \times 20$ for the single layer and bulk, respectively), $\epsilon_{\mathbf{k} n}$ are the band energies and $f_{\mathbf{k} n}$ the related

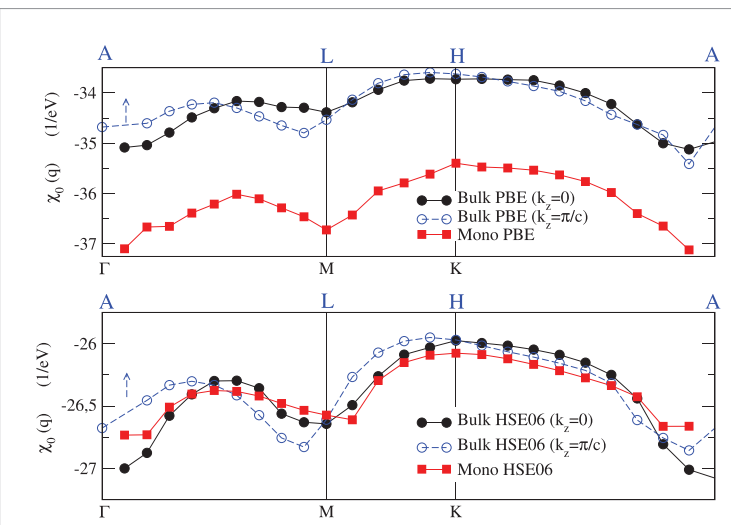

Figure 3. Calculated real part of $\chi_{0}(\mathbf{q})$ for bulk and monolayer $\mathrm{TiTe}_{2}$ using PBE and HSE06.

Fermi functions. Finally, $g_{\mathbf{k} n, \mathbf{k}+\mathbf{q} m}^{\nu}$ is the electronphonon matrix element for the mode $\nu$. The softening is then obtained as $\omega_{\mathbf{q} \nu}^{2}=\Omega_{\mathbf{q} \nu}^{2}+2 \Omega_{\mathbf{q} \nu} \prod_{\nu}^{\prime}(\mathbf{q})$.

In order to understand the mechanism responsible for the softening, we use maximally localized Wannier functions [39-41] and calculate the real part of the bare susceptibility with constant matrix elements, namely

$$
\chi_{0}(\mathbf{q})=\mathcal{P} \frac{1}{N_{k}} \sum_{\mathbf{k}, n m} \frac{f_{\mathbf{k} n}-f_{\mathbf{k}+\mathbf{q} m}}{\epsilon_{\mathbf{k} n}-\epsilon_{\mathbf{k}+\mathbf{q} m}}
$$

The quantity $\chi_{0}$ is related to $\prod_{\nu}^{\prime}(\mathbf{q})$ in the approximation of constant electron-phonon matrix elements, i.e. $g_{\mathbf{k} n, \mathbf{k}+\mathbf{q} m}^{\nu}=g$, as $\prod_{\nu}^{\prime}(\mathbf{q})=g \chi_{0}(\mathbf{q})$. Thus, it probes the effect of the electronic structure on the softening, but not those related to the dependence of the matrix element $g_{\mathbf{k} n, \mathbf{k}+\mathbf{q} m}^{\nu}$ on the exchange correlation functional or on $\mathbf{k}$ and band index. As it can be seen in figure 3 , the $\mathbf{q}$ dependence of $\chi_{0}(\mathbf{q})$ is very similar in the different cases (bulk and single layer), meaning that the main effect of the exchange functional on the harmonic spectra is due to a renormalization of the electron-phonon matrix elements and not of to a change in the electronic structure.

\subsection{Anharmonicity and charge ordering}

The calculations performed up to now neglect phonon-phonon scattering (anharmonicity) and its tendency to stabilize the lattice. In what follows, we carry out non-perturbative anharmonic calculations within the stochastic self-consistent harmonic approximation using HSE06 as the force engine. In particular, we evaluate the temperature dependent dynamical matrix

$$
\mathcal{D}=\left.\boldsymbol{M}^{-\frac{1}{2}} \frac{\partial F}{\partial \mathcal{R} \partial \mathcal{R}}\right|_{\mathcal{R}_{e q}} \boldsymbol{M}^{-\frac{1}{2}}
$$

where $\boldsymbol{M}$ is the matrix of the ionic masses $M_{a}$ with $M_{a b}=\delta_{a b} M_{a}$ and $\mathcal{R}$ are the coordinates of the centroids (i.e. average value of the atomic positions over the ionic wavefunction). The free energy and 


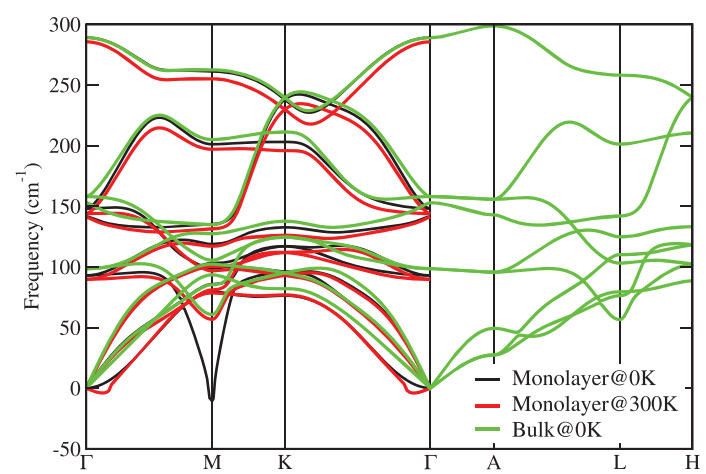

Figure 4. The anharmonic phonon dispersion of bulk (at $0 \mathrm{~K}$ ) and monolayer (at 0K and 300K) using the HSE06 functional from the CRYSTAL code.

Table 2. Distorted structure of single-layer $1 \mathrm{~T}-\mathrm{TiTe}_{2}$ within HSE06. The space group is P321 (number 150), as we use 3D labeling of space groups assuming an infinite distance between the $\mathrm{TiTe}_{2}$ layers. The Wyckoff positions are given as components with respect to the conventional cell. The in-plane lattice parameter is twice the experimental one of the $\mathrm{CdI}_{2}$ phase.

\begin{tabular}{lccc}
\hline atoms & $\mathrm{x}$ & $\mathrm{y}$ & $\mathrm{z}$ \\
\hline $\mathrm{Ti}$ & 0.0 & 0.0 & 0.0 \\
$\mathrm{Te}$ & -0.33311 & -0.16931 & 0.13099 \\
$\mathrm{Ti}$ & 0.49038 & 0.0 & 0.0 \\
$\mathrm{Te}$ & $1 / 3$ & $-1 / 3$ & -0.13034 \\
\hline
\end{tabular}

its second derivative can be obtained by performing appropriate stochastic averages over the atomic forces on supercells with ionic configurations obtained by displacing the atoms randomly from the equilibrium position and following a Gaussian distribution [1921]. While the free energy converges fairly quickly with the number of ionic configurations, the Hessian of the free energy is more noisy and a larger number of samples to converge. We use from $600-900$ and 400 force calculations for the single layer and bulk, respectively.

As the all-electron HSE06 force engine is computationally very expensive, we perform the calculation on a $4 \times 4$ supercell in the monolayer (i.e. 48 atoms containing the wavevector $\mathbf{q}=\mathbf{M}$ ) and a $4 \times 4 \times 2$ supercell in the bulk case (i.e. 96 atoms containing the wavevector $\mathbf{q}=\mathbf{L}$ ). We know from previous studies [18] that this supercell is large enough to describe charge density wave formation in the similar compound $1 \mathrm{~T}^{-} \mathrm{TiSe}_{2}$, but $\mathrm{T}_{\mathrm{CDW}}$ comes out somewhat underestimated (accurate determination of $\mathrm{T}_{\mathrm{CDW}}$ requires larger supercells, unaffordable with the HSE06 functional as the force engine). For this reason we perform calculations at zero and room temperature for the monolayer and at zero temperature for the bulk. We then determine if anharmonicity can stabilize or not the lattice. We expect that on larger supercells, the instability at the M point in the monolayer will be slightly stronger. More details on the

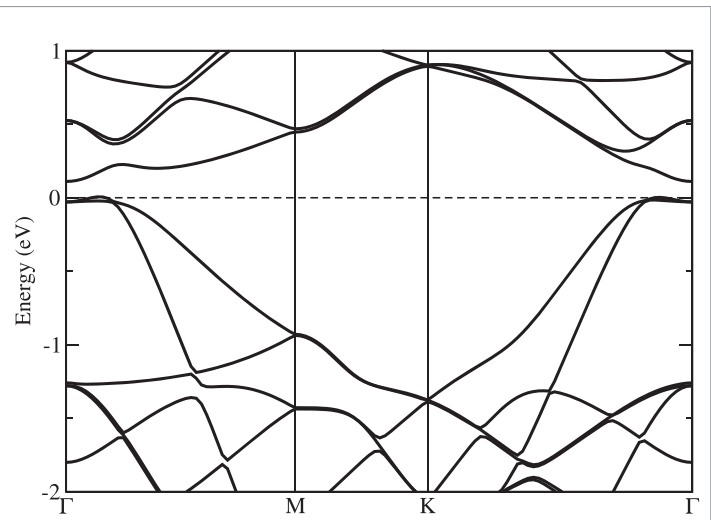

Figure 5. The HSE06 electronic band structure of monolayer $\mathrm{TiTe}_{2}$ in its low temperature phase $(2 \times 2$ superstructure).

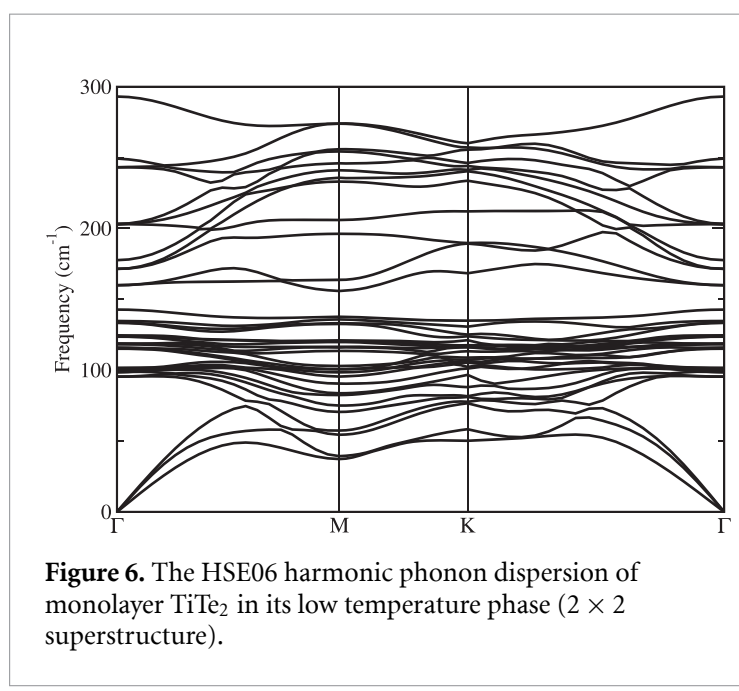

anharmonic calculation and the magnitude of the different terms occurring in equation (3) are given in appendix $\mathrm{C}$.

The results of the anharmonic calculation for the high- $T \mathrm{CdI}_{2}$ phase are shown in figure 4. As it can be seen, in both cases anharmonicity tends to stabilize the lattice and at $T=0 \mathrm{~K}$ the (positive) anharmonic correction to the soft mode at the $M$ point for the monolayer is similar in magnitude, to the corresponding one at the $\mathrm{L}$ point for the bulk. However, as the harmonic frequency is substantially much softer in the monolayer case, the anharmonic phonon frequency at the $\mathrm{M}$ point remains imaginary in the monolayer case, consistent with the experimental finding of a $2 \times 2$ reconstruction. On the contrary, in the bulk case, anharmonicity completely removes the imaginary phonon frequency at the $\mathrm{L}$ point, stabilizing the lattice and removing the charge ordering found at the harmonic level, again in agreement with the experimental findings [15]. At $T=300$ $\mathrm{K}$, the monolayer displays stable phonon frequencies. Thus, the thickness evolution of the CDW in $\mathrm{TiTe}_{2}$ is due to a competition of two effects, anharmonicity and the electron-phonon interaction. While anharmonicity has similar magnitude in bulk and single 
Table 3. Infrared active modes $\left(\mathrm{cm}^{-1}\right)$ in the CDW phase of $\mathrm{TiTe}_{2}$ single-layer and their decomposition in harmonic undistorted phonon modes. The point(s) $\mathbf{q}$ in the Brillouin zone and the frequency(s) $\omega_{\text {undist. }}$ label the frequencies and the phonon-momenta in the undistorted cell having the largest overlap with the zone phonon modes of the distorted cell. The number in parenthesis is the overlap in percentage (only those are bigger than $10 \%$ are shown).

\begin{tabular}{llll}
\hline Symmetry & HES06 (CDW phase $)$ & $\mathbf{q}$ & $\omega_{\text {undist. }}$ \\
\hline E & 79.46 & $\mathrm{M}$ & $82.52(50)+-102.09(25)+88.70(17)$ \\
A2 & 81.89 & $\mathrm{M}$ & $82.96(97)$ \\
$\mathrm{E}$ & 83.27 & $\mathrm{M}$ & $82.52(38)+88.70(23)+-102.09(18)$ \\
$\mathrm{E}$ & 91.79 & $\mathrm{M}+\Gamma+\mathrm{M}$ & $88.70(39)+101.17(25)+-102.09(15)$ \\
$\mathrm{E}$ & 104.09 & $\Gamma+\mathrm{M}$ & $101.2(29)+108.1(21)+-102.09(14)+108.87(14)$ \\
$\mathrm{A} 2$ & 105.54 & $\mathrm{M}$ & $108.99(81)$ \\
A2 & 106.84 & $\mathrm{M}$ & $108(67)+108.1(21)$ \\
$\mathrm{E}$ & 107.39 & $\mathrm{M}$ & $108.89(59)+108.87(17)+108.1(10)$ \\
$\mathrm{E}$ & 109.55 & $\Gamma+3 \mathrm{M}$ & $101.17(31)+108.08(27)+-102.09(15)+108.1(13)$ \\
$\mathrm{E}$ & 120.21 & $\mathrm{M}$ & $122.66(85)$ \\
$\mathrm{E}$ & 142.63 & $\Gamma$ & $143.8(92)$ \\
$\mathrm{E}$ & 172.79 & $\Gamma$ & $158.3(89)$ \\
A2 & 207.72 & $\mathrm{M}$ & $208.9(99)$ \\
$\mathrm{E}$ & 212.28 & $\mathrm{M}$ & $208.87(97)$ \\
A2 & 276.54 & $\mathrm{M}$ & $275.18(100)$ \\
$\mathrm{E}$ & 278.97 & $\mathrm{M}$ & $274.97(99)$ \\
A2 & 293.53 & $\Gamma$ & $290.04(99)$ \\
\hline
\end{tabular}

Table 4. Raman active modes $\left(\mathrm{cm}^{-1}\right)$ identified in the CDW phase of TiTe 2 monolayer. The point $(\mathrm{s}) \mathbf{q}$ in the Brillouin zone and the frequency(s) $\omega_{\text {undist. }}$ of the mode(s) of undistorted cell overlapping with the modes in the distorted cell are also reported, with the overlap percentage (only those are bigger than $10 \%$ are shown) between parentheses.

\begin{tabular}{llll}
\hline Symmetry & HES06 (CDW phase $)$ & $\mathbf{q}$ & $\omega_{\text {undist. }}$ \\
\hline E & 79.46 & $\mathrm{M}$ & $82.52(50)+-102.09(25)+88.70(17)$ \\
E & 83.27 & $\mathrm{M}$ & $82.52(38)+88.70(23)+-102.09(18)$ \\
A1 & 85.77 & $\mathrm{M}$ & $88.64(94)$ \\
$\mathrm{E}$ & 91.79 & $\mathrm{M}+\Gamma+\mathrm{M}$ & $88.70(39)+101.17(25)+-102.09(15)$ \\
$\mathrm{E}$ & 104.09 & $\Gamma+\mathrm{M}$ & $101.2(29)+108.1(21)+-102.09(14)+108.87(14)$ \\
$\mathrm{E}$ & 107.39 & $\mathrm{M}$ & $108.89(59)+108.87(17)+108.1(10)$ \\
$\mathrm{E}$ & 109.55 & $\Gamma+\mathrm{M}$ & $101.17(31)+108.08(27)+-102.09(15)+108.1(13)$ \\
A1 & 111.97 & $\mathrm{M}+\Gamma$ & $122.91(43)+-100.91(40)+145.96(11)$ \\
$\mathrm{E}$ & 120.21 & $\mathrm{M}$ & $122.66(85)$ \\
A1 & $\mathrm{M}+\Gamma+\mathrm{M}$ & $122.91(52)+145.96(27)+-100.91(18)$ \\
$\mathrm{E}$ & 130.72 & $\Gamma$ & $143.8(92)$ \\
A1 & 142.63 & $\Gamma$ & $144.71(89)+145.96(10)$ \\
A1 & 143.33 & $\Gamma+\mathrm{M}$ & $145.96(51)+-100.91(38)$ \\
$\mathrm{E}$ & 157.03 & $\Gamma$ & $158.3(89)$ \\
$\mathrm{E}$ & 172.79 & $\mathrm{M}$ & $208.87(96)$ \\
$\mathrm{E}$ & 212.28 & $\mathrm{M}$ & $274.97(99)$ \\
\hline
\end{tabular}

layer, the electron-phonon interaction leads to much more unstable harmonic phonons in the single layer at the $\mathrm{M}$ point than in the bulk at the $\mathrm{L}$ point. At the PBE level, however, the electron-phonon correction to the phonon frequency is not large enough to induce charge ordering. The HSE06 is responsible for a stronger electron-phonon interaction than in the PBE case. The HSE06 harmonic phonon dispersion displays CDWs both in bulk and in single layer, in disagreement with experiments. However, the single layer harmonic phonon frequencies are substantially more unstable than the ones in the bulk. Anharmonicity, similar in magnitude for the two case, removes the CDW in the bulk but not in the single layer, in perfect agreement with experiments.

\subsection{Structural, electronic and vibrational} properties of the single-layer charge ordered phase After explaining the appearance of CDW in singlelayer $\mathrm{TiTe}_{2}$, we study the low temperature $2 \times 2$ phase. Structural data for the single layer are given in table 2 obtained from geometrical optimization of forces and neglecting quantum effects. The distortion is analogous to that found in a single-layer $\mathrm{TiSe}_{2}$.

The HSE06 electronic structure of the distorted phase is shown in figure 5. An indirect gap of $0.1395 \mathrm{eV}$ is found to occur at the Fermi level. In STM experiments a pseudogap of $\approx 0.028 \mathrm{eV}$ at 42 $\mathrm{K}[14]$ is found in the CDW phase, substantially smaller. A similar overestimation of the gap in the low- $T$ phase by the HSE06 functional is found in single-layer 
$\mathrm{TiSe}_{2}$. Both these overestimations could be due to the neglect of nuclear quantum fluctuations in the low- $T$ phase and their consequences on the electronic gap.

In order to test the stability of the low- $T$ phase, we calculate the harmonic phonon dispersion using the HSE06 functional. The results are shown in figure 6 . We find dynamically stable phonon frequencies. As the Raman and infrared phonon frequencies can in future be used to determine the structural properties of the monolayer, we report in detail the zone center Raman and infrared modes in Tables. 4 and 3, respectively. Furthermore we report their decomposition in terms of the harmonic phonon modes of the undistorted structure, along the lines of what have been done in reference [17] (see supplemental materials in reference [17] for more details).

\section{Conclusions}

In this work we studied the $2 \mathrm{D}-3 \mathrm{D}$ crossover of the CDW transition in metallic $1 \mathrm{~T}-\mathrm{TiTe}_{2}$. This system is a remarkable exception between dichalcogenides as it shows no evidence of CDW formation in bulk, but it displays a stable $2 \times 2$ reconstruction in singlelayer form (most of metallic dichalcogenides display similar reconstructions in both bulk and single-layer form). In literature, the mechanism of the transition is unclear. Strain from the substrate and the exchange interaction have been pointed out as possible formation mechanisms. By performing non-perturbative anharmonic calculations with gradient corrected and hybrid functionals, we explained the thickness behaviour of the transition $1 \mathrm{~T}-\mathrm{TiTe}_{2}$. We first showed that, at the harmonic level, semilocal functionals fail in describing the CDW transition occurring in the monolayer, while the HSE06 functional predicts the occurrence of a CDW both in bulk and single layer, in disagreement with experiments. At the harmonic level, the presence of CDW at all thicknesses within HSE06 is not due to a change in the electronic structure but mostly to an exchange renormalization of the electron-phonon matrix element.

Our non-perturbative anharmonic calculations show that the occurrence of CDW in single-layer $\mathrm{TiTe}_{2}$ comes from the interplay of non-perturbative anharmonicity and an exchange enhancement of the electron-phonon interaction, leading to more unstable harmonic phonon modes in the single layer than in the bulk. Indeed, anharmonicity tends to stabilize both structure in a similar way, however, the larger instability present in the single layer at the harmonic level is not completely removed, while it is totally suppressed in the bulk.

Our calculations are for a suspended TiTe 2 monolayer. The presence of a substrate can in principle affect the behaviour of the CDW in the single layer. Assuming that the main effect of the substrate is mechanical stress and that no charge transfer occurs from the substrate to the $\mathrm{TiTe}_{2}$ single layer, two case can be conceived. If the lattice mismatching between the single layer and the substrate is large, then no in-plane mechanical strain is present and the results should be similar to the case of a suspended single layer. In the case of a close matching between the single-layer in-plane lattice parameter and the one of the substrate, then the net effect could be that of a compressive or tensile in-plane strain on the $\mathrm{TiTe}_{2}$ monolayer. The tensile stress tends to enhance the charge density wave while the compressive one to suppress it. However, in the measurements carried out so far, the lattice parameter of the supported monolayer is practically identical to the one of the bulk (see table 1), meaning that a negligible mechanical stress is present in the sample, further supporting the results of our work.

Finally, in an effort to better identify the properties of the single-layer $1 \mathrm{~T}^{-\mathrm{TiTe}_{2}} 2 \times 2 \mathrm{CDW}$ phase, yet not fully characterized experimentally, we study its electronic and structural properties and we provide a complete description of infrared and Raman active phonon modes in terms of the backfolding of the vibrational modes from the undistorted structure.

\section{Acknowledgments}

Computational resources were granted by PRACE (Project No. 2017174186) and from IDRIS, CINES and TGCC (Grant eDARI 91202 and Grand Challenge Jean Zay). M C, F M, J S Z and L M acknowledge support from the European Union's Horizon 2020 research and innovation programme Graphene Flagship under grant agreement No 881603.. M C and J S $\mathrm{Z}$ acknowledge support from Agence nationale de la recherche (Grant No. ANR-19-CE24-0028). F M and L M acknowledge support by the MIUR PRIN-2017 program, project number 2017Z8TS5B.

\section{Appendix A. Convergence tests}

In the main text, we have shown harmonic phonon calculations using well converged k-points mesh and Fermi-Dirac smearing that are summarized in table A1. Here in figure A1 we show the convergence of the lowest energy (unstable) phonon mode at $\mathrm{M}$ in the undistorted monolayer and at L using different functionals. In the table we list the converged parameters for all vibrational calculations.

\section{Appendix B. Relativistic effects and semilocal functionals.}

We show in figure B2 the effect of SOC on the gradient corrected electronic structure of bulk $\mathrm{TiTe}_{2}$. As it can be seen SOC is completely negligible in the monolayer and has slightly larger consequences in the bulk. In the bulk, however, SOC on top of gradient corrections fail 
(a) PBE monolayer

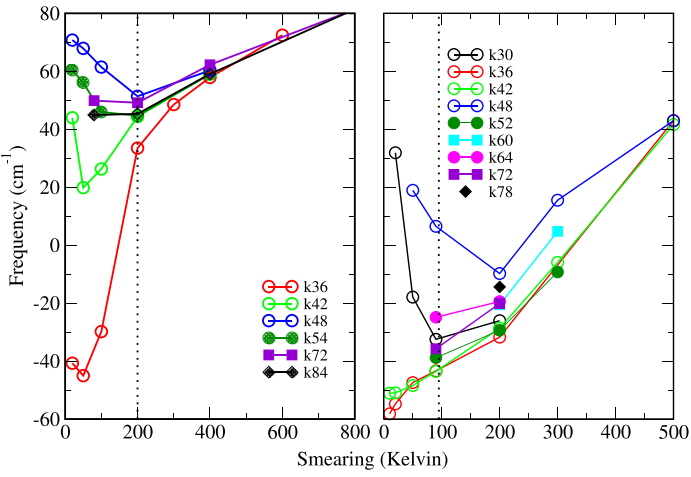

Figure A1. The convergence studies on the monolayer $\mathrm{TiTe}_{2}$ using PBE in its normal phase (a) and bulk $\mathrm{TiTe}_{2}$ using HSE06 (b). The vertical dotted line represents the converged smearing temperature. The legend shows different k-points mesh, e.g. k36 in (a) and (b) represents $36 \times 36 \times 1$ mesh and $36 \times 36 \times 8$, respectively. Note that in (b) we show the in-plane mesh convergence, and finally we converge the $k_{z}$ direction as concluded in table A1 .

Table A1. Converged parameters for the harmonic phonon calculations.

\begin{tabular}{lcc}
\hline methods & k-points & $\mathrm{T}_{e}$ (Kelvin) \\
\hline PBE (bulk) & $48 \times 48 \times 12$ & 315 \\
HSE06 (bulk) & $36 \times 36 \times 12$ & 95 \\
PBE (mono-HT) & $36 \times 36 \times 1$ & 200 \\
HSE06 (mono-HT) & $48 \times 48 \times 1$ & 32 \\
HSE06 (mono-LT) & $36 \times 36 \times 1$ & 30 \\
\hline
\end{tabular}

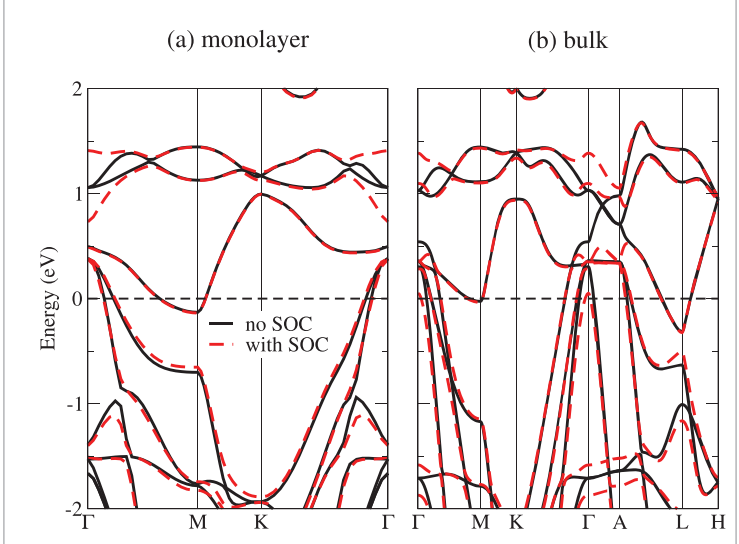

Figure B2. The effects of SOC in monolayer and bulk $\mathrm{TiTe}_{2}$ electronic band structures using PBE. Note the absence of a completely filled Te band at zone center (in disagreement with experiments) and in the HSE06 relativistic calculation in figure 1.

in reproducing the occurrence of a completely filled Te band at zone center (see figure 1 panels $(\mathrm{d}, \mathrm{e}, \mathrm{f})$ ). This failure is corrected by HSE06+SOC.

\section{Appendix C. different contributions to the free energy Hessian}

Here we provide a detailed analysis of all the different anharmonic terms contributing to the free energy Hessian. Within the SSCHA, the temperature dependent phonons are obtained from the dynamical matrix

$$
\mathcal{D}=\left.\mathbf{M}^{-\frac{1}{2}} \frac{\partial^{2} F}{\partial \mathcal{R} \partial \mathcal{R}}\right|_{\mathcal{R}_{e q}} \mathbf{M}^{-\frac{1}{2}}
$$

where $\mathbf{M}$ is the matrix of the ionic masses $M_{a}$ with $M_{a b}=\delta_{a b} M_{a}$, and $\left.\frac{\partial F}{\partial \mathcal{R} \partial \mathcal{R}}\right|_{\mathcal{R}_{e q}}$ is the free energy Hessian with respect to the centroid positions $\mathcal{R}$ reads [20]:

$$
\frac{\partial F}{\partial \mathcal{R} \partial \mathcal{R}}=\boldsymbol{\Phi}+\stackrel{(3)}{\mathbf{\Phi}} \boldsymbol{\Lambda}(0) \stackrel{(3)}{\mathbf{\Phi}}+\stackrel{(3)}{\mathbf{\Phi}} \boldsymbol{\Lambda}(0) \boldsymbol{\Lambda}(0) \stackrel{(3)}{\mathbf{\Phi}}
$$

where $\boldsymbol{\Phi}$ represents the SSCHA force constant, (3) (3)

$\boldsymbol{\Phi} \Lambda(0) \Phi$ is the so-called 'static bubble term', and $\stackrel{(3)}{\boldsymbol{\Phi}} \boldsymbol{\Lambda}(0) \boldsymbol{\Lambda}(0) \stackrel{(3)}{\boldsymbol{\Phi}}$ contains the higher order terms. Here $\stackrel{(n)}{\Phi}$ refers to the $n$th order anharmonic force constants averaged over the density matrix of the SSCHA hamiltonian (see reference [20] for more details on notation). All these quantities can be recasted as appropriate stochastic averages over the atomic forces. The corresponding dynamical matrix can be written as:

$$
\mathcal{D}=\stackrel{2}{\mathcal{D}}+\mathcal{D}^{\text {bubble }}+\mathcal{D}^{\text {other }},
$$

where

$$
\begin{gathered}
\stackrel{2}{\mathcal{D}}=\mathbf{M}^{-\frac{1}{2}} \boldsymbol{\Phi} \mathbf{M}^{-\frac{1}{2}} \\
\mathcal{D}^{\text {bubble }}=\mathbf{M}^{-\frac{1}{2}} \stackrel{(3)}{\mathbf{\Phi}} \boldsymbol{\Lambda}(0) \stackrel{(3)}{\mathbf{\Phi}} \mathbf{M}^{-\frac{1}{2}} \\
\mathcal{D}^{\text {other }}=\mathbf{M}^{-\frac{1}{2}} \stackrel{(3)}{\mathbf{\Phi}} \boldsymbol{\Lambda}(0) \boldsymbol{\Lambda}(0) \stackrel{(3)}{\boldsymbol{\Phi}} \mathbf{M}^{-\frac{1}{2}}
\end{gathered}
$$

Analogously, with $\stackrel{2}{\mathcal{D}}$ o we refer to the harmonic dynamical matrix:

$$
\stackrel{2}{\mathcal{D}} o=\left.\mathbf{M}^{-\frac{1}{2}} \frac{\partial^{2} \mathrm{~V}}{\partial \mathbf{R} \partial \mathbf{R}}\right|_{\mathbf{R}_{0}} \mathbf{M}^{-\frac{1}{2}}
$$

where $\left.\frac{\partial V}{\partial \mathbf{R} \partial \mathbf{R}}\right|_{\mathbf{R}_{0}}$ is the Born-Oppenheimer potential energy Hessian in the 'classical' equilibrium configuration $\mathbf{R}_{0}$. The function $\boldsymbol{\Lambda}(0)$ (See equation (22) in reference [20]) is mainly determined by the eigenvectors and eigenvalues of $\stackrel{2}{\mathcal{D}}$. The different contributions to the dynamical matrix are shown in figure C3. As it can be seen, the contributions arising from $\mathcal{D}^{\text {bubble }}$ and $\mathcal{D}^{\text {other }}$ are negligible for the bulk case. In the monolayer, $\mathcal{D}^{\text {other }}$ is negligible, however $\mathcal{D}^{\text {bubble }}$ is non negligible and it is the term responsible for the 


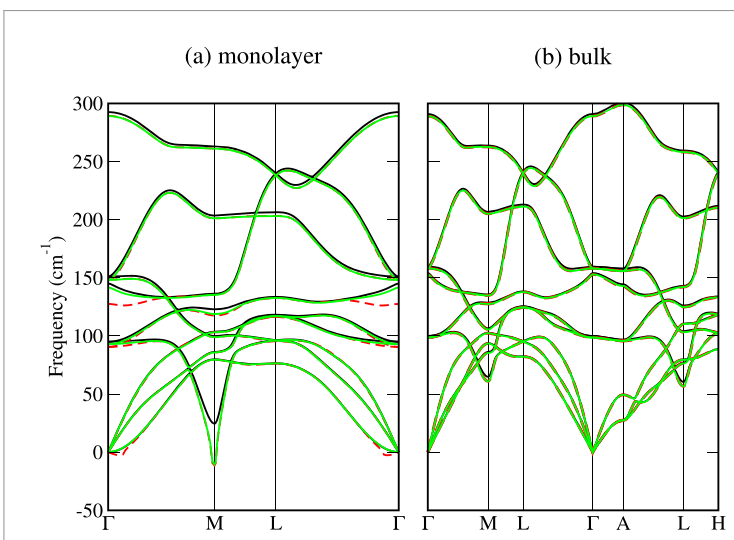

Figure C3. The individual contribution in SSCHA beyond the harmonic approximation at $0 \mathrm{~K}$. Black, red and green

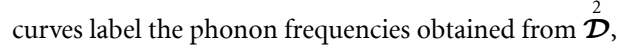
$\stackrel{2}{\mathcal{D}}+\mathcal{D}^{\text {bubble }}$ and $\stackrel{2}{\mathcal{D}}+\mathcal{D}^{\text {bubble }}+\mathcal{D}^{\text {other }}$, respectively
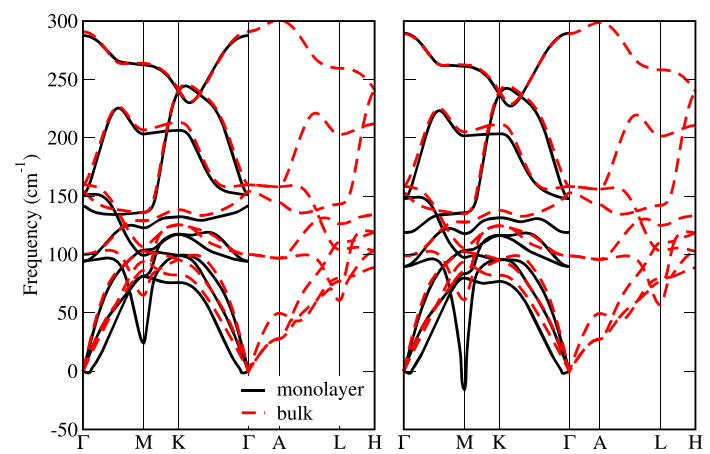

Figure C4. The phonon frequencies calculated using $\stackrel{2}{\mathcal{D}}$ (left panel) and $\stackrel{2}{\mathcal{D}}+\mathcal{D}^{\text {bubble }}$ (right panel) for monolayer and bulk $\mathrm{TiTe}_{2}$ at $0 \mathrm{~K}$.

occurrence of the charge density wave. A clearer comparison between the $\stackrel{2}{\mathcal{D}}$ and $\mathcal{D}^{\text {bubble }}$ in bulk and monolayer is shown in figure $\mathrm{C} 4$, underlining again the role of $\mathcal{D}^{\text {bubble }}$ in the monolayer.

\section{ORCID iD}

Matteo Calandra (1) https://orcid.org/0000-00031505-2535

\section{References}

[1] Wilson JA, Di Salvo FJ and Mahajan S 1975 Adv. Phys. 24 117-201

[2] Novoselov K S, Jiang D, Schedin F, Booth T J, Khotkevich V V, Morozov S V and Geim A K 2005 Proc. Natl Acad. Sci. 102 10451-3

[3] Stan R M, Mahatha S K, Bianchi M, Sanders C E, Curcio D, Hofmann P and Miwa J A 2019 Phys. Rev. Mater. 3044003

[4] Lin H, Huang W, Zhao K, Lian C, Duan W, Chen X and Ji S H 2018 Nano Res. 114722

[5] Kolekar S, Bonilla M, Ma Y, Diaz H C and Batzill M 2018 2D Mater. 5015006
[6] Wang $\mathrm{H}$ et al 2018 Adv. Mater. 301704382

[7] Li L J, O’Farrell E C T, Loh K P, Eda G, Özyilmaz B and Castro Neto A H 2016 Nature 529 185-9

[8] Duong D L, Ryu G, Hoyer A, Lin C, Burghard M and Kern K 2017 ACS Nano 11 1034-40

[9] Sugawara K, Nakata Y, Shimizu R, Han P, Hitosugi T, Sato T and Takahashi T 2016 ACS Nano 10 1341-5

[10] Chen P, Chan Y H, Fang X Y, Zhang Y, Chou M Y, Mo S K, Hussain Z, Fedorov A V and Chiang T C 2015 Nat. Commun. 68943

[11] Fang X Y, Hong H, Chen P and Chiang T C 2017 Phys. Rev. B 95 (20) 201409

[12] Sakabe D, Liu Z, Suenaga K, Nakatsugawa K and Tanda S 2017 npj Quantum Mater. 222

[13] Pásztor A, Scarfato A, Barreteau C, Giannini E and Renner C 2017 2D Mater. 4041005

[14] Chen P et al 2017 Nat. Commun. 8516

[15] Guster B, Robles R, Pruneda M, Canadell E and Ordejón P 2018 2D Materials 6015027

[16] Fragkos S, Sant R, Alvarez C, Bosak A, Tsipas P, Tsoutsou D, Okuno H, Renaud G and Dimoulas A 2019 Adv. Mater. Interfaces 61801850

[17] Hellgren M, Baima J, Bianco R, Calandra M, Mauri F and Wirtz L 2017 Phys. Rev. Lett. 119176401

[18] Zhou J S, Monacelli L, Bianco R, Errea I, Mauri F and Calandra M 2019 arXiv:1910.12709

[19] Errea I, Calandra M and Mauri F 2013 Phys. Rev. Lett. 111177002

[20] Bianco R, Errea I, Paulatto L, Calandra M and Mauri F 2017 Phys. Rev. B 96014111

[21] Errea I, Calandra M and Mauri F 2014 Phys. Rev. B 89064302

[22] Monacelli L, Errea I, Calandra M and Mauri F 2018 Phys. Rev. B 98024106

[23] Perdew J P, Burke K and Ernzerhof M 1996 Phys. Rev. Lett. 77 3865-8

[24] Heyd J, Scuseria G E and Ernzerhof M 2003 J. Chem. Phys. 118 8207-15

[25] Krukau A V, Vydrov O A, Izmaylov A F and Scuseria G E 2006 J. Chem. Phys. 125224106

[26] Dovesi R et al 2018 Wiley Interdiscip. Rev.: Comput. Mol. Sci. 8 e1360

[27] Giannozzi P et al 2009 J. Phys.: Condens. Matter. 21395502

[28] Giannozzi P et al 2017 J. Phys.: Condens. Matter. 29465901

[29] Weigend F and Ahlrichs R 2005 Phys. Chem. Chem. Phys. 7

[30] Heyd J, Peralta J E, Scuseria G E and Martin R L 2005 J. Chem. Phys. 123174101

[31] Peterson K A, Figgen D, Goll E, Stoll H and Dolg M 2003 J. Chem. Phys. 119 11113-23

[32] van Setten M, Giantomassi M, Bousquet E, Verstraete M, Hamann D, Gonze X and Rignanese G M 2018 Computer Phys. Commun. 226 39-54

[33] Patel S and Balchin A 1985 J. Mater. Science. Lett. 4 382-4

[34] Claessen R et al 1996 Phys. Rev. B 54 2453-65

[35] de Boer D K G, van Bruggen C F, Bus G W, Coehoorn R, Haas C, Sawatzky G A, Myron H W, Norman D and Padmore H 1984 Phys. Rev. B 29 6797-809

[36] Rajaji V et al 2018 Phys. Rev. B 97085107

[37] Strocov V N, Krasovskii E E, Schattke W, Barrett N, Berger H, Schrupp D and Claessen R 2006 Phys. Rev. B 74195125

[38] Calandra M, Profeta G and Mauri F 2010 Phys. Rev. B 82165111

[39] Mostofi A, Yates J, Pizzi G, Lee Y, Souza I, Vanderbilt D and Marzari N 2014 Comput. Phys. Commun. 1852309

[40] Marzari N and Vanderbilt D 1997 Phys. Rev. B 56 12847-65

[41] Souza I, Marzari N and Vanderbilt D 2001 Phys. Rev. B 65035109 